\title{
Kinematic Analysis on the Serving Technique of Tennis Athlete Roushwali
}

\author{
Yu Gu and Jihe Zhou \\ Department of Chengdu Sport Institute, Chengdu 610041, China
}

\begin{abstract}
This report is to analyze the serve technique of elite tennis athlete Roushwali, the champion of 2017 ITF Junior Masters. The report discussed this topic in virtue of three-dimensional video analytic method. According to the purpose and need for this study and combined with the actual technical movements, the serving technology could be divided into three stages as follows: throwing ball rising racket stage, backward swing stage and forward swing stage, to acquire the outstanding youth male tennis players' kinematic analysis for each stage is conducted. The results show that the serving technology of Roushwali is reasonable and it is worthwhile to establish a sport model.
\end{abstract}

Keywords-tennis serves technique; kinematic; three-dimensional video analysis;modeling

\section{RESEARCH PURPOSES}

The 2017 ITF Junior Masters has been ended at the Sichuan International Tennis Center on October 29, 2017. In the men's singles final, Finnish teenager Roushwali beat the Chinese player Wu Yi-Bing on the tie-break by a neck and won the Men's singles champion. In this paper, the three-dimensional cinema is been used for making a kinematical analysis to get the data when Roushwali is serving a ball. Not only to explore the serving technical model of excellent young tennis players, but also to provide the technical serving data for coaches and athletes.

\section{STUdy OBJECT AND METHOD}

\section{A. Study Object}

Finnish male tennis player Emir Roushwali, champion of the men's singles at the 2017 ITF Junior Masters.

\section{B. Study Method}

Three-dimensional video analysis: Erection of two cameras at the scene of the game, shooting athletes from different angles shot technology, shooting frequency was 50 frames / second. By using 3D Signal Tec software to shoot video data analysis, Dempster human body model was used when analyzed. According to the needs of the study, the tennis racket and the tennis ball were added when parsing two test points. The resulting data is smoothed using a low-pass filter with a cut-off frequency of $8 \mathrm{~Hz}$.

\section{STUDY RESULTS}

According to the needs of research and analysis, combined with the actual technical action, the service technology is divided into three stages: There are throwing ball rising racket stage that is when the ball in the hand falling to the lowest point when left knee bent to the maximum; backward swinging stage, that is the left knee flexion was the most to the lowest point in time behind shot; and forward swinging hit stage, that is, the racket after the lowest point in time to hit the ball.

\section{A. Throwing Ball rising Racket Stage}

When the ball in the hand falling to the lowest point, left knee was bended to the maximum. The purpose of this action phase is to throw the ball steady in the air at a predetermined position, creating a favorable condition for the subsequent stroke action phase.

The height when the ball throws away, that is also the ball away from the ground. Roushwali throw away from the height of $2.12 \mathrm{~m}$, the ball away from the compare to his height ratio is 1.03. Related research found that throwing the ball when the ball away from a reasonable height should be in the head ${ }^{[1]}$, that the height of the ball away from the height ratio should be slightly greater than 1 , the analysis that a higher ball away from hand makes control distance more longer, so the height of throwing ball compared to hand serving the ball is lower, the ball is more stable, more able to throw the ideal position.

At the time of the ball's leaving, the left elbow joint angle, the left shoulder joint angle and the left hip joint angle of Roushwali were $161.5^{\circ}, 96.7^{\circ}$ and $165.9^{\circ}$ respectively, and the values of three joint angles were all smaller than the general figures. Generally, when throwing the ball in the stage of throwing the elbow joint angle greater stretching more fully, the ball thrown more stable; shoulder angle should not be neither too large nor small, the angle too big will let the ball falling far from the body, the angle too small will let the ball falling too close to the body, they are all not conducive for hitting; the greater the angle of the hip joint, the more fully stretch of the top hip, the more conductive to the formation of a better back bow posture.

At this stage the athlete's lower legs' flexion and squat, the purpose is to provide a good starting position and strength for the "scratch back" action. In the lower limb flexion process, knee extensor elastic energy stored. Roushwali at this stage left knee minimum flexion angle of $102.3^{\circ}$, the angle of right knee joint was $133.1^{\circ}$. It analysis the depth of the body when the knee the size of the knee can affect the next stage of the movement range, Roushwali knee flexion at this moment sufficient to facilitate the next phase of the action development.

\section{B. Backward Swing Stage}

Backward swing phase, that is from the maximum knee flexion time to beat in the lowest point of time after the 
movement process. In this stage the racquet will be left behind, the body form an effective force forward "back bow" gesture, commonly known as "scratch back" action.

Knee extension of the left knee, that is, the maximum flexion of the left knee and knee extension at the moment when the end of the range between the left knee angle. The maximal flexion angle of Roushwali left knee joint was $107.9^{\circ}$, the left knee joint angle was $153.8^{\circ}$, the range at the end of pedaling was $45.9^{\circ}$. The maximal pedaling knee extension velocity was $244.6^{\circ} / \mathrm{s}$. Studies have shown that the rapid kicking of the knee can form a better back bow, which accumulates sufficient potential energy for subsequent actions. At this stage, Roushwali the amplitude kicks was fully.

Right elbow flexion angle can reflect the swing back to the magnitude of the action, the greater the amplitude, the longer the swing distance, swing speed faster. At this stage, the minimum angle of the right elbow joint of Roushwali is $72.1^{\circ}$, which shows that Roushwali has a large elbow range and a long swing distance, which is sufficient for swinging forward ready.

\section{Forward Swing Stage}

Swing the ball forward stage is from the racket after the lowest point in time to hit the ball. The purpose of this stage is to get the maximum speed of the racket to hit the ball.

In the process, the height of Roushwali's center of gravity increased, the racket hit the ball, and the height is $2.98 \mathrm{~m}$. Some studies suggest that the height and height of batting point ratio of about 1.5 times more appropriate ${ }^{[10]}$, Roushwali's hit point height compare to his height ratio was 1.45 , so the shot height is reasonable.

At the same time, experts point out that the gap between the highest point of throwing and the striking point should be about $0.5 \mathrm{~m}^{[1]}$. The difference value between the highest point of the throwing and the striking point is $0.45 \mathrm{~m}$; within it should be a reasonable range. Swing distance, is also the lowest point of the racket to the vertical distance between the hitting point, the distance of Roushwali was $1.8 \mathrm{~m}$, swing range is sufficient. And the greater of the swing distance, the distance of accelerate longer, the faster the ball hit.

Roushwali serve a successful score.

\section{STUDY CONCLUSIONS}

The following conclusions are drawn from the data analysis of the serve video taken by 3D photograph analytic method.

During the throwing ball rising racket stage, the ball deviate from Roushwali `s hand on the high is 2.12 meters, the ratio of height to height is 1.03; At the moment that the angel of left elbow, left shoulder, left hip are $161.5^{\circ}, 96.7^{\circ}, 165.9^{\circ}$. Lower extremity flexion squatting moment, Roushwali's left and right knees joints minimum flexion angle were $121.7^{\circ}, 145.4^{\circ}$.For the consistency of the height to throw the ball, Roushwali has a great control.

During the backward swing stage, Roushwali `s left knee joints largest flexion was $153.8^{\circ}$,the angel of pedal and stretch was $45.9^{\circ}$,the largest stretch angular velocity was $244.6^{\circ} / \mathrm{s}$, the minimum angle of the right elbow was $72.1^{\circ}$. Roushwali was full stretch and can save a lot of potential for the subsequent swing.

During the forward swing stage, the height of the hitting point is $2.98 \mathrm{~m}$, the ratio of hitting points to height is 1.45 , the difference between the highest point and the hit point is $0.45 \mathrm{~m}$. All are able to hit the ball effectively within a reasonable range.

Roushwali's serve technical is worthwhile to establish a sport model.

\section{REFERENCES}

[1] Chuanjia Du,Jihe Zhou, Wangshuai. A study on the stability of serving technique for elite young tennis players[C].CABS,2016.

[2] Yirong Li,Jihe Zhou. A comparative analysis of the service technology of Enquist and Moya based on the 3d virtual action reconstruction system[J].Journal of Chengdu Technological University,2015.

[3] Chuanjia Du,Jihe Zhou. Excellent tennis player dimitrov serves the technology of 3D virtual reproduction kinematics analysis[C].The 10th National Convention on Sport Science of China,2015.

[4] Huifang Wang,Jihe Zhou. Analysis of the service technique of the male champion of ATP tennis chengdu open[C].CABS,2012.

[5] Beixiang Liu. Sports biomechanics (sports technology analysis and evaluation[M]. Chengdu:Sichuan Science and Technology Press,2008.

[6] Botao Yan,Yanxi Wu,Tinggang Yuan.Biomechanical analysis of tennis serve technology [J]. CHINA SPORT SCIENCE,2000,20(4):80-85.

[7] Cuilan Zhou,Chengren Zhang. A brief discussion on the tactics and tactics of tennis[J].Tennis World,1997(4):20.

[8] Liwei Han,Chunyan Li. A brief analysis of sport biomechanics of tennis serve technology[J].Contemporary Sports Technology,2013.

[9] Jingxu Fan,Jixue Yuan. A review of the biomechanics of tennis serve technique[J].Sports World Scholarly,2017.

[10] Zhangnan. Biomechanical analysis of tennis serve technology[J].Science Park.2012.3:4 